\title{
Top-N Recommendation based on Mutual Trust and Influence
}

\author{
D.W. Seng, J.X. Liu, X.F. Zhang, J. Chen, X.J. Fang
}

\section{Dewen Seng*}

School of Computer Science and Technology, Hangzhou Dianzi University

Hangzhou 310018, China

*Corresponding author: sengdw@163.com

\section{Jiaxin Liu}

School of Computer Science and Technology, Hangzhou Dianzi University Hangzhou 310018, China

\section{Xuefeng Zhang}

School of Computer Science and Technology, Hangzhou Dianzi University Hangzhou 310018, China

\section{Jing Chen}

School of Computer Science and Technology, Hangzhou Dianzi University Hangzhou 310018, China

\section{Xujian Fang}

School of Computer Science and Technology, Hangzhou Dianzi University Hangzhou 310018, China

\begin{abstract}
To improve recommendation quality, the existing trust-based recommendation methods often directly use the binary trust relationship of social networks, and rarely consider the difference and potential influence of trust strength among users. To make up for the gap, this paper puts forward a hybrid top-N recommendation algorithm that combines mutual trust and influence. Firstly, a new trust measurement method was developed based on dynamic weight, considering the difference of trust strength between users. Secondly, a new mutual influence measurement model was designed based on trust relationship, in light of the social network topology. Finally, two hybrid recommendation algorithms, denoted as FSTA(Factored Similarity model with Trust Approach) and FSTI(Factored similarity models with trust and influence), were presented to solve the data sparsity and binarity. The two algorithms integrate user similarity, item similarity, mutual trust and mutual influence. Our approach was compared with several other recommendation algorithms on three standard datasets: FilmTrust, Epinions and Ciao. The experimental results proved the high efficiency of our approach.

Keywords: mutual trust, mutual influence, social recommendation system, cold start, data sparsity.
\end{abstract}

\section{Introduction}

The continuous expansion of e-commerce provides users with direct access to a growing number and variety of products. In this case, the users need to spend a lot of time looking for their desired products. This poses a huge challenge to information consumers and producers. To cope with the challenge, it is necessary to recommend the information and products favored by the users according to their interest features and purchase behavior, that is, to set up a recommendation system. For the users, the recommendation system enables them to pinpoint desired information out of a massive amount of data; for the merchants, the system helps to decide 
on which products to sell to a group of users and provide satisfactory services that enhance user loyalty [14].

Collaborative filtering recommendation is an important way of personalized recommendation, thanks to its ability to discover new interest points of users and a dazzling array of resources [4]. However, this recommendation approach is bottlenecked by problems like cold start and data sparsity in its algorithms. How to alleviate the two problems with the social attributes of the users has become the main task in the design of the recommendation system [17]. Among the possible solutions, the recommendation algorithm combined with social information, i.e., the social recommendation algorithm, comes as an effective means to solve the said problems [14]. The social information main includes the mutual trust and influence between users, both of which are essential to social activities [15].

The mutual trust has long been recognized as the key to recommendation system $[1-3,8-$ $10,12,14,15,23]$. In such a system, the rating can be predicted more accurately by replacing or supplementing the rate-based similarity [3]. The trust-based recommendation can effectively suppress the cold start problem and improve the accuracy and coverage of the recommendation [16]. In fact, trust communication has been proved as a social science, the key to social network analysis and an important phenomenon in recommendation scenarios [10]. But the existing measure of trust is only represented by 0 and 1 binary $[8,9]$, and there is no clear value to indicate the specific trust between users. Hence, This paper not only improves the recommendation effect, but also alleviates the sparseness of social network data through the specific measurement of trust between users. In addition, through the study of social networks, we have found a new inspiration, what is the trust in social networks? In the traditional recommendation system, few people combine mutual trust and mutual influence. But in social networks, user influence really exists and has a certain impact on the recommendations between users [15].

In light of the above analysis, this paper attempts to find out the social relationship data that guide the recommendation, disclose and weight the effect of the guidance on user preference, and improve the results of the social recommendation system. For these purposes, two new hybrid recommendation algorithms were proposed for top- $\mathrm{N}$ recommendation, based on the factorized similarity model based on trust (FST). The two algorithms are respectively denoted as FSTA and FSTI. The innovations of our research are summed up as follows:

(1) Based on dynamically weighted trust relationship, a new mutual trust measurement method was put forward according to the difference in mutual trust strength. The method takes account of the direct and indirect trust relationships between users, thus improving the recommendation accuracy.

(2) Using the topology of social network, a new mutual influence measurement model was developed based on trust relationship. Considering both direct and indirect mutual influences, the proposed model makes full use of the implicit information in trust relationship.

(3) Two new hybrid top-N recommendation models, involving user similarity, item similarity, mutual trust and mutual influence, were designed to solve the data binarity and sparsity of mutual trust, and used to explore the existence of social network users, identify potential trust relationships, and set up a mutual trust network.

(4) Our models were proved efficient through repeated comparative verifications on three standard datasets, namely, FilmTrust, Epinions and Ciao. 


\section{Literature review}

Social recommendation refers to the construction of a social relationship network between users based on the additional input of their social relationship information [20]. As long as the network users have direct or indirect social relationships, it is possible to generate proper recommendations for new users based on the interest models of the known users [17]. A collaborative filtering algorithm that combines social relationships is called a social recommendation algorithm. Many scholars and researchers have probed deep into the theories, methods and applications of social recommendation systems $[10,20,23,24]$. Below is a brief review of some representative studies.

To improve the accuracy of rating prediction, Fang et al. [3] replaced and supplemented rate-based similarity in recommendation systems with trust. Wang and Ma [18] considered and weighted user's rating and trust similarity. Moradi and Ahmadian [12] proposed a trust-based singular value decomposition technique, TrustSVD, which evaluates both the explicit and implicit effects of trust. The above studies show that the inclusion of trust can enhance the accuracy of the recommendation system. On this basis, it is assumed that the recommendation accuracy can be improved through the combination of trust and other important social factors.

The advent of social networks has shortened the distance and enhanced the links between people, providing an excellent experimental platform and a huge amount of data for impact research [8]. Taking this golden opportunity, many researchers have analyzed and simulated the mutual influence in many social networks, and achieved fruitful results with huge application values [21,25]. For instance, Li et al. [15] developed social recommendation algorithms through collaborative filtering, based on mutual trust and influence. With its important impact on user behavior in social networks, the mutual influence should be fully considered in the identification of influential users, such as to enhance the performance and accuracy of the recommendation system.

The personalized top- $\mathrm{N}$ recommendation system has a direct bearing on many real-world applications, such as e-commerce platforms and social networks [22]. However, the existing top- $\mathrm{N}$ recommendation methods become less effective with the growth in data sparsity. This problem can be solved by introducing social information like mutual trust and preference to the recommendations system. In this way, it is possible to mine out the implicit information from the social network, and overcome the defect of sparse datasets.

\section{Recommendation based on mutual trust and influence}

This section introduces mutual trust and influence to the social recommendation method of the FST, aiming to improve the effectiveness of traditional collaborative filtering. The twomodule structure of the FST model combining the mutual trust and influence is shown in Figure 1 , where the blue dashed lines indicate that a trusted user has an influence on the truster, and the black dotted lines indicate user $u$ 's rating of item $j$. In the left half of the model, user $u$ trusts and is influenced by user $v$, who has rated item $j$ as $r(v, j)$; the goal is to predict user $u$ 's rating $r(u, j)$ for item $j$, considering the mutual trust and influence between the two users. In the right half of the model, user $k$ trusts and is influenced by user $u$, and has rated item $j$ as $\left.r_{(} k, j\right)$; the rating may affect user $u$ 's rating $\left.r_{(} u, j\right)$ for the same item $j$.

Following this model, the social network relationship and a user's item ratings are analyzed to predict the item ratings of the other user. Finally, the item set with the higher rating is recommended to the users. Let $U=u_{1}, u_{2}, \cdots, u_{m}, I=i_{1}, i_{2}, \cdots, i_{n}$ and $R=\left[r_{u, i}\right]_{m \times n}$ be the set of $m$ users, the set of $n$ items, and the matrix of item ratings in the rating-based collaborative filtering algorithm, where $r_{u, i}$ is user $u$ 's rating of item $i$ (the rating is generally a real number 


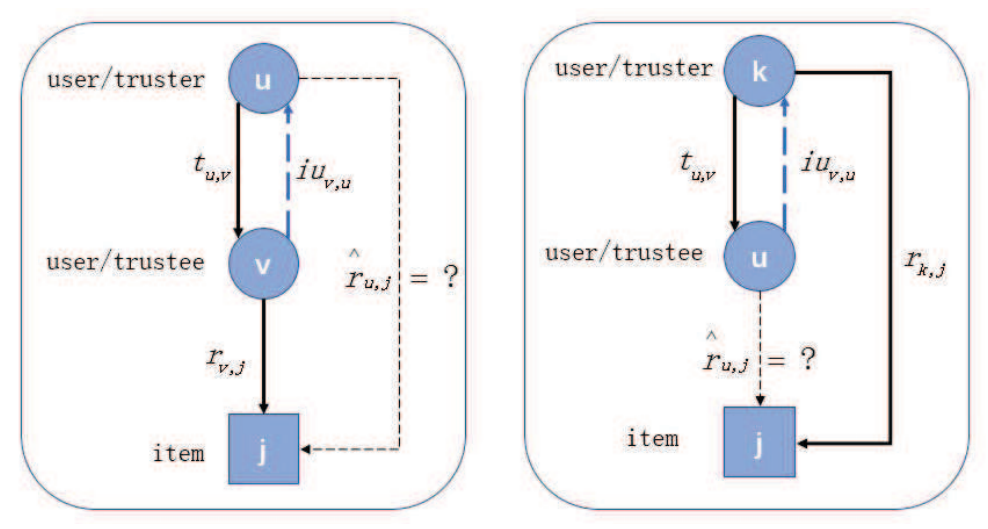

Figure 1: FST model combining mutual trust and influence

between $[0,5]$, and positively correlated with the user's interest in the item). The set of items rated by user $u$ and the set of users having rated item $i$ are respectively expressed as $I_{u}=$ $i \mid r_{u, i} \neq 0$ and $U_{i}=u \mid r_{u, i} \neq 0$.

In a social network, the users' mutual trust can be represented by $T=\left[t_{u, v}\right]_{m \times m}$, where $t_{u, v}$ is the trust between $u$ and $v$; the users' mutual influence can be represented by $I U=\left[i u_{u, v}\right]_{m \times m}$, where $i u_{u, v}$ is the influence between $u$ and $v$. Note that each user in the social network has $N$ friends.

\subsection{Mutual trust ranking}

If taken as the auxiliary information, mutual trust, an important type of social information, can effectively solve data sparsity problem in collaborative recommendation algorithms. In realworld applications, however, there following problems still exists: (1) The trust relationship is too sparse to be measured accurately; (2) The trust relationship is often expressed in the binary form (0 or 1$)$, failing to weigh the exact trust strength between users.

\section{Dynamically weighted trust measurement model}

This subsection explores the rating prediction based on user's social relationship and constructs a model for mutual trust measurement. To begin with, the two users, $A$ and $B$, in the social network are assumed to have only one interaction, i.e. rated the same item. During the interaction, each user has an impact on the other's trust if both of them have given the same rating. The success of the interaction hinges on the rating difference between the two users. The interaction process is shown in Figure 2, where the uppercase letters in circular boxes are items and the lowercase letters in rectangular boxes are users. It can be seen that, both users $A$ and $B$ have rated the same item $a$, i.e. engaged in an interaction. If the two users' ratings on the item is not above the threshold $\epsilon$, then the interaction is successful; otherwise, the interaction is a failure.

Mutual trust is the accumulation of subjective feelings of individuals on each other. For two users $u$ and $v$, the greater user $u$ trusts user $v$, the more its interactions with $v$. The initial mutual trust $\operatorname{Init}(u, v)$ can be defined as:

$$
\operatorname{Init}(u, v)=\frac{\min \left(I_{u} \bigcap I_{v}, D\right)}{D}
$$

where $I_{u} \cap I_{v}$ is the number of interactions between the two users, i.e. the number of items rated the same by the two users; $D$ is an adjustable threshold that measures the minimum 


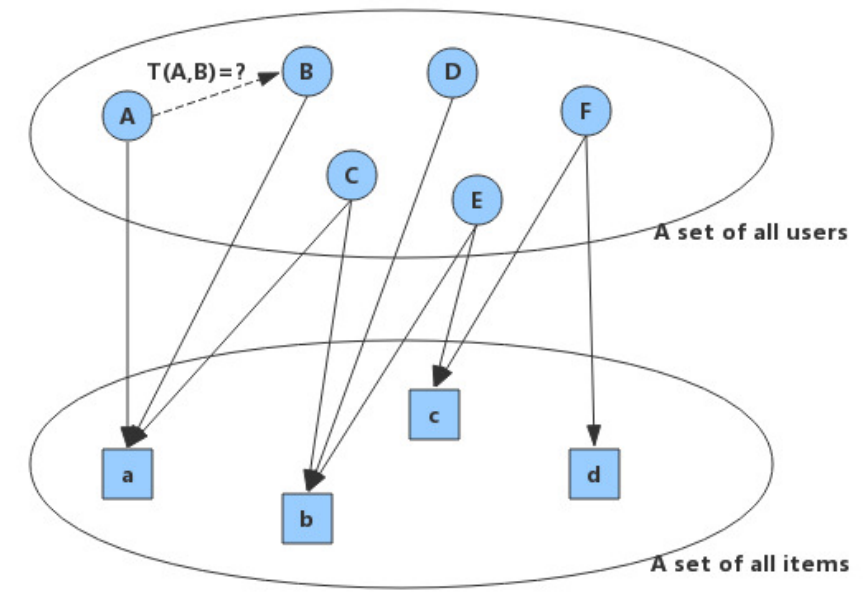

Figure 2: The interaction between users

number of interactions when two users fully trust each other. If $I_{u} \cap I_{v}$ is not greater than $D$, then the effective weight will come into force; otherwise, the initial strength of the mutual trust will be set to 1 . Since the users differ in the number of ratings, the criterion for full trust may vary with users. Thus, the threshold for each user can be set to $D_{u}=\sqrt{\left|I_{u}\right|}$.

Next, the preference of user $A$ for item $c$ can be defined as [19]:

$$
P(A, c)=\frac{\sum_{m} \in A_{c} \operatorname{sim}(A, m)}{\left|A_{c}\right|}
$$

where, $A_{c}$ is to the set of $\mathrm{m}$ users having rated item $c$. Equation (2) shows that the preference of the users in set $A_{c}$ for item $c$ increases with their similarity with user $A$.

Since each pair of users will optimize the mutual trust through interaction, the trust between the two users $T(U, V)$ can be calculated by assigning a difference weight to the item based on the difference in the two users' preferences for the same item:

$$
T(A, B)=\left\{\begin{array}{cc}
\operatorname{IniT} D(A, B) \frac{\sum_{c \in \text { success }} P(A, c)-\sum_{c \in \text { failure }} P(A, c)}{\sum_{c \in \text { success }} P(A, c)+\sum_{c \in \text { failure }} P(A, c)} & , T(A, B) \\
0 & \text { Otherwise }
\end{array}\right.
$$

where, Init $(u, v)$, the initial trust between the two users is a dynamic weighting factor. Obviously, the fewer commonly rated items, the smaller the numerator, and the less the initial trust will contribute to the final mutual trust. The trust of each user to the other users can be computed by equation (3), and a set of trusted users can be obtained by filtering the results against the threshold.

\section{FSTA model}

The rating $r_{u, i}$ of user $u$ on item $i$ was predicted based on mutual trust, item similarity and user similarity. The mutual trusts of each target user with its trustee(s) and truster(s) were obtained by equation (3). After the calculation, the list of trust users was updated, and the implicit information in the user rating matrix was obtained to obtain the directed trust between users. The item similarity was the inner product of two lower-order matrices $X$ and $Y$, where $X \in R^{n \times d}$ and $Y \in R^{n \times d}$. Note that $d<<n$ is the number of potential feature vectors associated 
with the item. The user similarity was obtained from two lower-order matrices $P \in R^{m \times d}$ and $Q \in R^{m \times d}$, where $d<<m$ is the number of potential feature vectors associated with the user.

Then, the ratings were ranked to produce a set of recommended items for each target user. The ratings of the user can be predicted as:

$$
\begin{aligned}
\widehat{r}_{u, i} & =b_{i}+s\left|U_{i-u}\right|^{-\beta} \sum_{v \in U_{i-u}} p_{v}^{T} q_{u}+(1-s)\left|I_{u-i}\right|^{-\alpha} \sum_{j \in I_{u-i}} x_{j}^{T} y_{j} \\
& +\sigma\left|T_{u}^{+}\right|^{-z} \sum_{a \in T_{u}^{+}} p_{a}^{T} y_{i}+(1-\sigma)\left|T_{u}^{-}\right|^{-\chi} \sum_{b \in T_{u}^{-}} p_{b}^{T} y_{i}
\end{aligned}
$$

where, $\alpha, \beta, z$ and $\chi \geq 0$ are the number of rated items, that of similar users, that of trustees and that of trusters; $b_{i}$ is the bias of item $i ; s$ and $\delta \in[0,1]$ are user similarity and the relative importance of trust, respectively. For each pair of trustee $a \in T_{u}^{+}$and truster $b \in T_{u}^{-}$, the inner products $p_{a}^{T} y_{i}$ and $p_{b}^{T} y_{i}$ describe the influences of users $a$ and $b$ on the trust of the target user, respectively.

\subsection{Mutual influence ranking}

By mining the information in the trust relationship, the author put forward a method to calculate the implicit influence between users, taking account of both the direct and indirect trust relationships between users.

\section{Influence calculation}

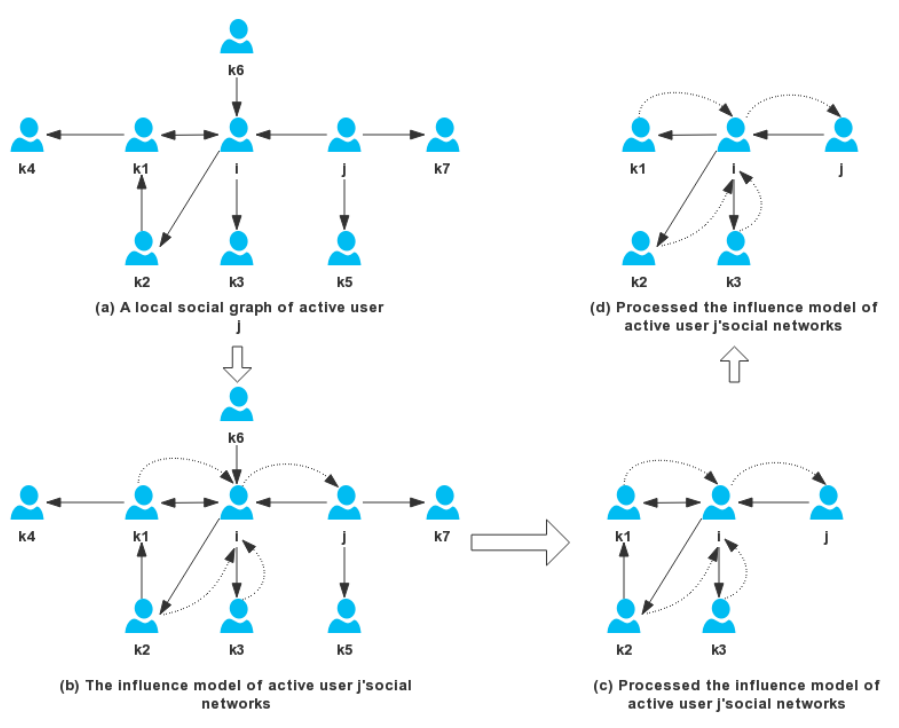

Figure 3: Local social network

As shown in Figure 3, the local social network consists of 9 users, respectively denoted as $i, j, k 1, k 2, k 3, k 4, k 5, k 6$ and $k 7$. The goal is to estimate the trust strength between users $i$ and $j$. The trust strength cannot be determined accurately if only the set of trusts (solid lines in Figure 3(a)) is considered. To fully exploit the information in the trust relationship, a trust measurement model was designed based on dynamic weight. By definition, user $j$ is affected by 
user $i$ when it trusts that user. Then, the nodes with no influence on node $j$ were removed. After that, the trust relationships with no influence were deleted from the network.

Inspired by the above research, an improved algorithm was proposed to identify the influence of users in the trust network.

Definition 1. In a mutual trust network $G_{T}$, the edge weight $w_{i} j$ can be defined as the trust strength between users $i$ and $j$, that is, $w(i, j)=T(i, j)$. The nodes and directed edges in the network respectively stand for users and strengths between users.

Definition 2. The weight of node $w(i)$ can be interpreted by the trustee of the mutual trust network as $w(i)=\sum_{j \in T_{i}^{+}} w_{i j}$.

Definition 3. The relative importance of node $\mathrm{j}$ in the eyes of its neighbor node $\mathrm{i}$ can be calculated by $p(i, j)=w_{i, j} / w(i)$. If node $\mathrm{j}$ or its neighbor node $\mathrm{k}$ are of high relative importance, then node i will invest more time and energy in node $\mathrm{j}$. This reflects the fact that people often devote more time and energy to those with great importance or importance relationships.

Definition 4. In light of the features of mutual trust network, an improved model was designed to measure mutual influence $C(i)$ :

$$
C(i, j)=\left\{\begin{array}{r}
p(i, j)+\sum_{k \in T_{i}^{+}} p(i, j) p(i, k), C(i, j) \geq v_{c} \\
0, \text { Otherwise }
\end{array}\right.
$$

where, $p(i, j)$ and $\sum p(i, j) p(i, k)$ are the direct and indirect influences of user $i$ on user $j$, respectively. The latter is determined by the number of intermediate nodes $k$ between node $i$ and node $j$. It can be seen from equation (5) that the value of $C(i, j)$ is positively correlated with the number of trust neighbors of each node and the closeness between network nodes.

\section{FSTI model}

To predict each user's rating of an item, it is assumed that user $u$ in the above FSTA model is affected by a set of users $I U_{u}=\left\{w \mid i u_{u, w}\right\}$. On this basis, another social recommendation algorithm FSTI was constructed based on the implicit influence of mutual trust.

Firstly, the item rating was modelled by matrix decomposition. The rating prediction formula can be derived from the results of equation (5) and the mutual influence matrix as:

$$
\begin{aligned}
\widehat{r}_{u, i} & =b_{i}+s\left|U_{i-u}\right|^{-\beta} \sum_{v \in U_{i-u}} p_{v}^{T} q_{u}+(1-s)\left|I_{u-i}\right|^{-\alpha} \sum_{j \in I_{u-i}} x_{j}^{T} y_{j} \\
& +\delta\left|T_{u}^{+}\right|^{-z} \sum_{a \in T_{u}^{+}} p_{a}^{T} y_{i}+(1-\delta)\left|T_{u}^{-}\right|^{-\chi} \sum_{b \in T_{u}^{-}} p_{b}^{T} y_{i}+\left|I U_{u}\right|^{-\theta} \sum_{w \in I U_{u}} p_{w}^{T} y_{i}
\end{aligned}
$$

where, $\theta \geq 0$ is the number of users that influences user $u$ (hereinafter referred to as influential users); $I U_{u}$ is the set of influential users; $s$ and $\delta$ are the control parameters; $p_{w}^{T} y_{i}$ is the inner product equal to the influence of each influential user $w \in I U_{u}$ over user $u$.

Next, the variables $b, P, Q, X$ and $Y$ can be computed by the following objective function:

$$
J=\frac{1}{2} \sum_{u \in C} \sum_{i \in I_{u}^{+}, j \in I_{u}^{-}}\left\|\left(r_{u, i}-r_{u, j}\right)-\left(\widehat{r}_{u, i}-\widehat{r}_{u, j}\right)\right\|^{2}+\frac{\lambda}{2}\left(\|P\|_{F}^{2}+\|Q\|_{F}^{2}+\|X\|_{F}^{2}+\|Y\|_{F}^{2}+\|b\|_{F}^{2}\right)
$$


where, $C$ is the set of all users; $I_{u}^{+}$is the set of items rated by user $u ; I_{u}^{-}$is the set of items not rated by user $u ;\|\cdot\|_{F}^{2}$ is the Frobenius norm.

For convenience, the regularization parameter $\lambda$ was employed to process all the relevant variables. The recommendation effect can be greatly improved through proper adjustment and assignment of these variables.

Compared with the FST, the FSTI has the following features:

(1) The FSTI replaces the trust matrix $T$ with the trust strength estimation matrix $S$, rather than assume that a user has the same trust strength with any friend;

(2) The FSTI estimates the mutual influence matrix $I U$ by the implicit influence of trust relationship, revealing the implicit mutual influence;

(3) The FSTI improves the recommendation accuracy through the comprehensive consideration of item similarity, user similarity, mutual trust and mutual influence.

\subsection{Model learning and complexity analysis}

\section{FSTA complexity}

First, the gradients and update rules for all variables were calculated for model training (lines 3-44, Algorithm 1). All variables were initialized with a random value in $(0,0.01)$ (line 1). In each iteration, the overall computing cost in Algorithm 1 was approximately $O(n b)$, where $\mathrm{n}$ is the number of ratings in the training set and $b$ is the mean number of users evaluating the item, and the sampling factor $\rho$ (line 5) was used to randomly sample a set of negative instances of $Z$ to train the model. The variables were repeatedly updated by the stochastic gradient descent (SGD) rules (lines 16-44) until the loss value had converged or the maximum number of iterations had been reached. Next, the converged user potential feature matrix $P$ and the item implicit feature matrix $X$ were outputted, marking the end of the training (line 45). Finally, all learned variables were returned as outputs.

\section{FSTI complexity}

The FSTI follows basically the same training procedure as the FSTA. To save space, the details on the implementation of the algorithm are omitted here. The pseudocode of the FSTI is listed in Algorithm 2. The trained model was used to predict each user's ratings of unknown items, and the items with the highest ratings were collected into a recommendation set.

\section{Summary of FSTA and FSTI algorithm complexity}

Referring to Algorithm 1, the FSTA time complexity of our method mainly includes the evaluation of the objective function and the calculation of the gradient of each variable. For each iteration, the total computation time cost of Algorithm 1 is $O\left(n_{t} b d(|R|+|T|)\right)$, where $n_{t}$ is the number of training matrices, $b$ represents the average number of graded items for the user, and $d$ represents the feature vector dimension. Relatively speaking, FSTI increases the influence of influence on the basis of FSTA, so the time complexity of FSTI is $O\left(n_{t} b d(|R|+|T|+|I U|)\right)$. Since the rating matrix $R$ and the trust matrix $T$ and the influence matrix $I U$ are very sparse, the time complexity of our method FSTI is much lower than the matrix cardinality. The above analysis shows that our strategy for personalized recommendation successfully integrates user similarity, mutual trust and mutual influence. 


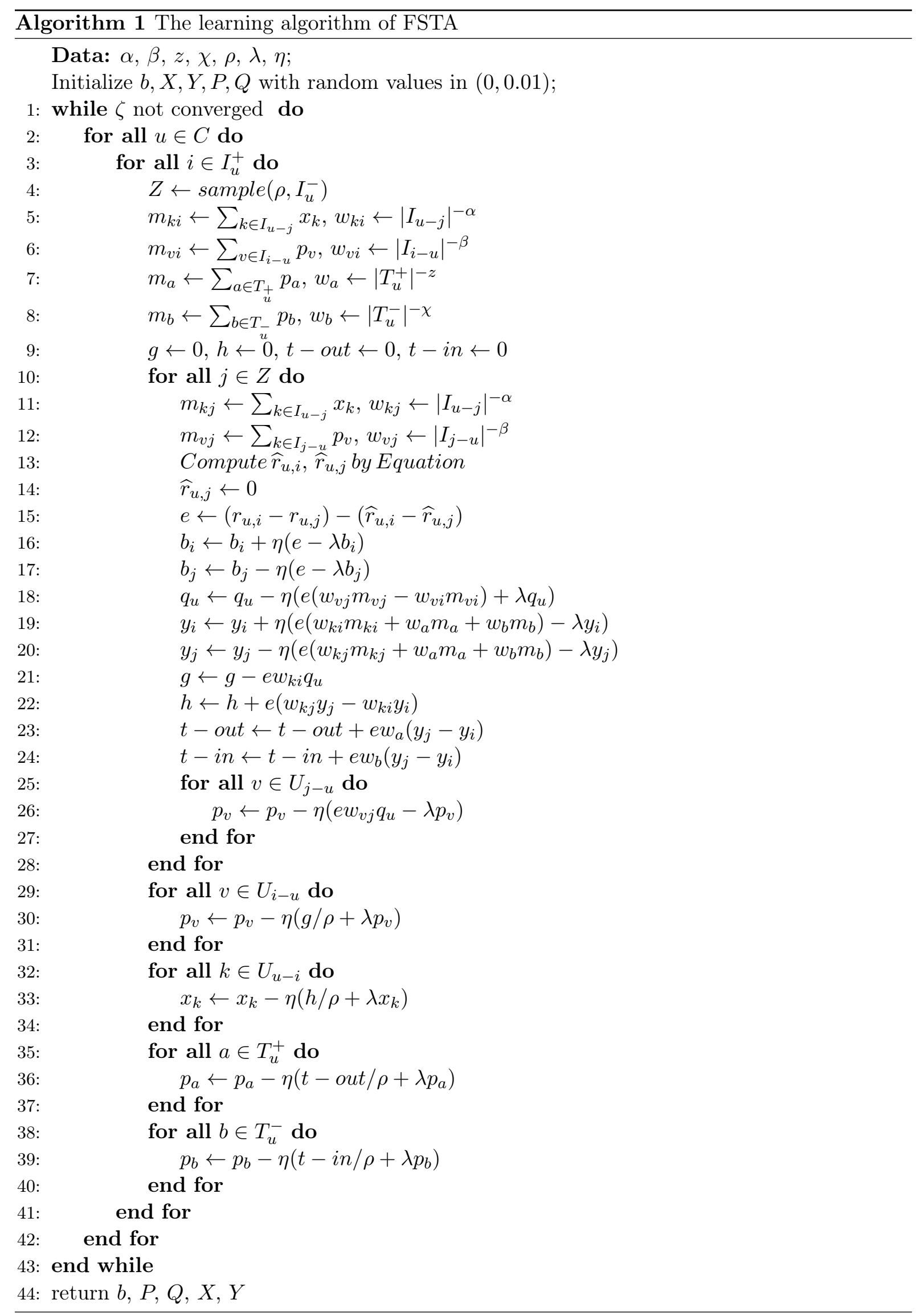




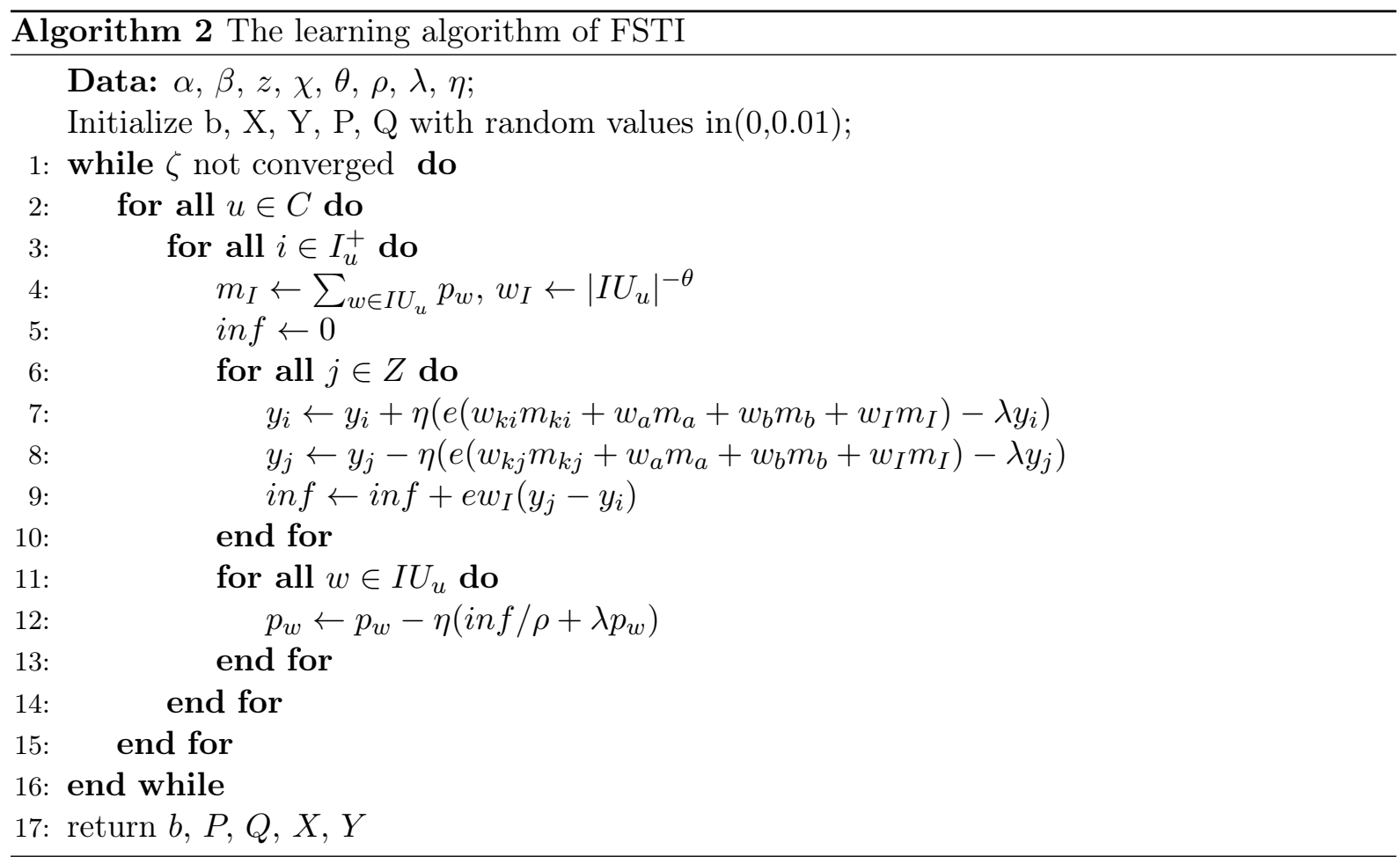

\section{Verification and results}

This section verifies the parameter robustness and sensitivity in our strategy through several experiments on three public datasets, and compares the effectiveness of our strategy with six top$\mathrm{N}$ recommendation methods based on implicit feedbacks.

\subsection{Experimental setup}

\section{Datasets}

The experiment data include three datasets, namely, Flimtrust, Ciao, and Epinions [4]. Each dataset contains ratings and mutual trust of users in social network. Upon signup, each user was allowed to rate all items, and browse the ratings by other users to make more favorable decisions. In addition, the user can befriend any trusted user, forming a network of trust relationships. For the datasets Epinions and Ciao, the rating was given against a five-point scale, where 1 means strongly dislike and 5 means strongly like. The mutual trust was described in binary form: existence (1) and absence (0). Our approach was applied to the three datasets to estimate the potential mutual trust and make accurate recommendations. As shown in Table 1, all the three datasets are extremely sparse, except FilmTrust.

Table 1: Datasets

\begin{tabular}{cccc}
\hline Data Set & FilmTrust & Ciao & Epinions \\
\hline Users & 1,508 & 7,375 & 40,163 \\
Items & 2,071 & 99,746 & 139,738 \\
Ratings & 35,479 & 278,483 & 664,824 \\
Density & $1.14 \%$ & $0.0379 \%$ & $0.0118 \%$ \\
\hline
\end{tabular}




\section{Comparative methods}

Our approach was compared with the following six top-N recommendation methods based on implicit feedbacks:

(1) BPR: Bayesian personalized ranking. Based on implicit feedback, the BPR is extended from the top- $\mathrm{N}$ ranking recommendation algorithm by the pairing hypothesis for item ordering, using implicit feedback, Matrix Factorization (MF) and K-Nearest Neighbors (KNN) model [19].

(2) GBPR: The BPR based on group preference. The GBPR is an improved version of the BPR with richer interaction between users [16].

(3) MostPop: The MostPop is the baseline method that ranks the ratings of an item by popularity, i.e. how frequently the item is rated or consumed by the user.

(4) FISM: Factored item similarity model. The FISM alleviates the sparsity of the existing top$\mathrm{N}$ recommendation algorithm by taking the product of two low-dimensional latent factor matrices as the similarity matrix [11] .

(5) FST: Factored Similarity model with Trust + . The FST introduces the mutual trust matrix and user similarity matrix into the FISM, thereby alleviating the sparsity of existing top-N recommendation algorithm and enhancing the accuracy of ranking recommendation [9].

(6) FSTA, FSTI: It is our proposed method for comparing two methods. Among them, FSTI adds the influence of user influence on the basis of FSTA.

\section{Evaluation indices}

The previous studies $[14,19]$ on recommendation systems have shown that the common error rate indices of rating prediction, such as the mean square error (MSE) and root mean square error (RMSE), cannot fully characterize the performance of the recommendation algorithm. In fact, the algorithm performance mostly depends on the recommendation of the top-N items. To fully verify the effect of our approach, the FSTI was combined with the BPR for top-N recommendation [19], and evaluated the hit rate by precision, recall rate and F1-measure:

$$
P @ N=\frac{1}{\left|U^{\prime}\right|} \sum_{u \in U^{\prime}} \frac{\left|R_{N}(u) \cap I_{u}^{\prime}\right|}{N} R @ N=\frac{1}{\left|U^{\prime}\right|} \sum_{u \in U^{\prime}} \frac{\left|R_{N}(u) \cap I_{u}^{\prime}\right|}{I_{u}^{\prime}} F 1 @ N=\frac{2 \cdot P @ N \cdot R @ N}{P @ N+R @ N}
$$

where, $I_{u}^{\prime}$ is the set of items overestimated by user $u$; $U^{\prime}$ is the test set; $P @ N, R @ N$ and $F 1 @ N$ are the precision, recall rate and $F 1-$ measure under $N$ recommended items, respectively. Sometimes, the $P @ N$ and $R @ N$ may contradict each other. In this case, the $F 1-$ measure should be taken into account to solve the contradiction. The greater the values of $P @ N$ and $F 1 @ N$, the better the recommendation effect.

The five-fold cross validation was adopted in our experiments. Each dataset was split randomly into 5 parts. In each iteration, 4 parts were allocated to the training set and the remaining one to the test set. The mean results of the 5 parts were taken as the final results. The number of recommended items was selected in 5,10 .

Note that common evaluation indices like mean absolute error (MAE) and RMSE are not applicable to our research, because they are mainly used to evaluate prediction performance rather than the top- $\mathrm{N}$ recommendation effect. 


\section{Parameter settings}

The parameters in our experiments were configured empirically, e.g. the number of potential factors was set to $d=10$. For the GBPR, the number of users in the set was fixed as 5 and the parameters were adjusted by the sampling factor $\rho \in[0,1]$. For all BPR-based methods, the unsorted items for model learning were selected through uniform sampling [19]. For all decomposition-based models, parameters $\alpha, \beta, z, \chi$ and $\theta$ were selected from a set of small values, i.e. $\{0.5,1,2\}$, and the sampling factor $\rho$ was fixed at 10 (Algorithm 1), for the FISM satisfies the interval of $[11,17]$. For all matrix-based methods, the setting of the regularization parameter $\lambda$ was optimized in $\{0.000001,0.00001,0.0001,0.01,0.1\}$ by grid search.

\subsection{Effect of parameters $\alpha, \beta, z, \chi$ and $\theta$}

The five parameters $\alpha, \beta, z, \chi$ and $\theta$ describes item similarity, user similarity, mutual trust and mutual influence. In our experiments, the value of each parameter is adjusted in the small set of $\{0.5,1,2\}$ when the control parameters and were 0.5 . Tables 2 and 3 respectively list the parameter configurations corresponding to the five best $P @ 5$ results of the FSTA and the FSTI on each dataset. Obviously, the different parameter configurations led to different results, and the optimal parameters changed from dataset to dataset.

Table 2: The optimal parameter configurations of the FSTA on the three datasets.

\begin{tabular}{|c|c|c|c|c|c|c|c|c|c|c|c|c|}
\hline Methods & \multicolumn{4}{|c|}{ Filmtrust } & \multicolumn{4}{|c|}{ Ciao } & \multicolumn{4}{|c|}{ Epinions } \\
\hline Parameters & $\alpha$ & $\beta$ & $z$ & $\chi$ & $\alpha$ & $\beta$ & $\chi$ & $\chi$ & $\alpha$ & $\beta$ & $\chi$ & $\chi$ \\
\hline \multirow[b]{2}{*}{1} & 2 & 0.5 & 0.5 & 0.5 & 0.5 & 2 & 0.5 & 0.5 & 0.5 & 1 & 0.5 & 1 \\
\hline & \multicolumn{4}{|c|}{0.4192} & \multicolumn{4}{|c|}{0.02892} & \multicolumn{4}{|c|}{0.01222} \\
\hline \multirow{2}{*}{2} & 0.5 & 2 & 0.5 & 1 & 2 & 1 & 2 & 1 & 2 & 0.5 & 2 & 2 \\
\hline & \multicolumn{4}{|c|}{0.4187} & \multicolumn{4}{|c|}{0.02843} & \multicolumn{4}{|c|}{0.01194} \\
\hline \multirow{2}{*}{3} & 0.5 & 2 & 2 & 0.5 & 2 & 0.5 & 1 & 2 & 2 & 2 & 0.5 & 0.5 \\
\hline & \multicolumn{4}{|c|}{0.4186} & \multicolumn{4}{|c|}{0.02821} & \multicolumn{4}{|c|}{0.01177} \\
\hline \multirow{2}{*}{4} & 0.5 & 1 & 0.5 & 0.5 & 1 & 1 & 0.5 & 2 & 0.5 & 0.5 & 1 & 0.5 \\
\hline & \multicolumn{4}{|c|}{0.4184} & \multicolumn{4}{|c|}{0.02808} & \multicolumn{4}{|c|}{0.01159} \\
\hline \multirow{2}{*}{5} & 0.5 & 1 & 0.5 & 1 & \multirow{2}{*}{\multicolumn{4}{|c|}{0.02804}} & 1 & & 1 & 2 \\
\hline & \multicolumn{4}{|c|}{0.4184} & & & & & \multicolumn{4}{|c|}{0.01158} \\
\hline
\end{tabular}

Table 3: The optimal parameter configurations of the FSTI on the three datasets.

\begin{tabular}{|c|c|c|c|c|c|c|c|c|c|c|c|c|c|c|c|}
\hline Methods & \multicolumn{5}{|c|}{ Filmtrust } & \multicolumn{5}{|c|}{ Ciao } & \multicolumn{5}{|c|}{ Epinions } \\
\hline Parameters & $\alpha$ & $\beta$ & $z$ & $\chi$ & $\theta$ & $\alpha$ & $\beta$ & $z$ & $\chi$ & $\theta$ & $\alpha$ & $\beta$ & $z$ & $\chi$ & $\theta$ \\
\hline \multirow{2}{*}{1} & 0.5 & 0.5 & 2 & 1 & 2 & 0.5 & 1 & 2 & 1 & 0.5 & 1 & 2 & 2 & 0.5 & 1 \\
\hline & \multicolumn{5}{|c|}{0.4198} & \multicolumn{5}{|c|}{0.02953} & \multicolumn{5}{|c|}{0.01260} \\
\hline \multirow{2}{*}{2} & 0.5 & 2 & 2 & 0.5 & 1 & 0.5 & 1 & 1 & 0.5 & 2 & 1 & 0.5 & 0.5 & 2 & 0.5 \\
\hline & \multicolumn{5}{|c|}{0.4194} & \multicolumn{5}{|c|}{0.02941} & \multicolumn{5}{|c|}{0.01233} \\
\hline \multirow{2}{*}{3} & 1 & 2 & 1 & 2 & 0.5 & 2 & 1 & 0.5 & 1 & 0.5 & 0.5 & 0.5 & 2 & 1 & 2 \\
\hline & \multicolumn{5}{|c|}{0.4192} & \multicolumn{5}{|c|}{0.02932} & \multicolumn{5}{|c|}{0.01215} \\
\hline \multirow{2}{*}{4} & 1 & 0.5 & 2 & 1 & 0.5 & 2 & 2 & 2 & 0.5 & 2 & 0.5 & 2 & 3 & 1 & 0.5 \\
\hline & \multicolumn{5}{|c|}{0.4172} & \multicolumn{5}{|c|}{0.02930} & \multicolumn{5}{|c|}{0.01214} \\
\hline \multirow{2}{*}{5} & 0.5 & 1 & 0.5 & 1 & 2 & \multirow{2}{*}{\multicolumn{5}{|c|}{$\begin{array}{c}1 \\
0.029 \\
\end{array}$}} & 1 & 2 & 1 & 1 & 0.5 \\
\hline & & & 0.419 & & & & & & & & \multicolumn{5}{|c|}{0.0120} \\
\hline
\end{tabular}

The experimental results of the FSTA corresponding to parameters $\alpha, \beta, z$ and $\chi$ are recorded in Tables 4 , and denoted by $P @ 5$. It can be seen that the results were optimal at $\alpha=1, \beta=2$ 
and $z>0.5$. As a result, before making top- $\mathrm{N}$ recommendations, the item similarity, the trustee and the truster should be emphasized over user similarity. The experimental results of the FSTI corresponding to parameters $\alpha, \beta, z, \chi$ and $\theta$ are recorded in Tables 5 , and denoted by $P @ 5$. It can be seen that the results were optimal at $\alpha=1, \beta=2$ and $z>0.5$ and $\theta<2$. As a result, before making top- $\mathrm{N}$ recommendations, the item similarity, the trustee and the truster should be emphasized over user similarity.

\subsection{Effect of control parameter $\mathrm{s}$}

For each dataset, the parameters $\alpha, \beta, z, \chi$ and $\theta$ were set to their optimal values, the first control parameter $\delta$ was fixed at 0.5 , and the second control parameter $s$ was adjusted in the interval $[0,1]$ by a step size of 0.1 . The effects of the adjustment on the FSTA and the FSTI are displayed in Figure 4(a) and Figure 4(b), respectively.

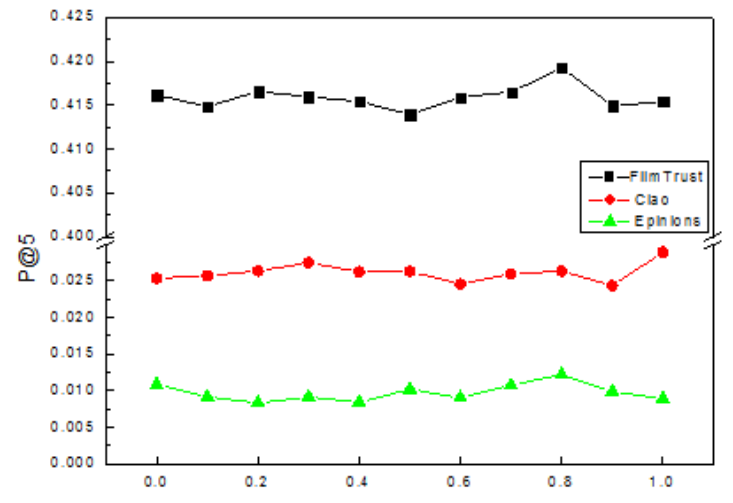

(a) FSTA

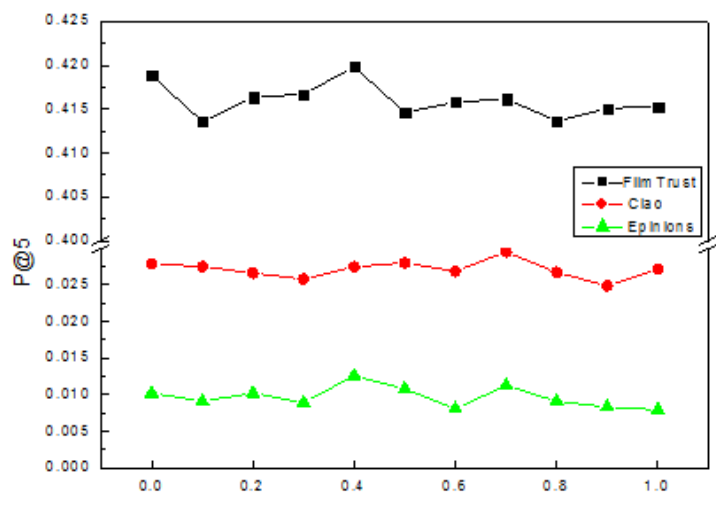

(b) FSTI

Figure 4: The effect of parameter $s$ on our approaches FSTA and FSTI in terms of precision at 5

As shown in Figure 4, the optimal $s$ values of the FSTA on FilmTrust, Ciao and Epinions were $0.8,1$ and 0.8 , respectively, revealing that, in the FSTA model, user similarity has a stronger promotion effect on top- $\mathrm{N}$ recommendation accuracy than item similarity. Meanwhile, the optimal $s$ values of the FSTI on FilmTrust, Ciao and Epinions were 0.4, 0.7, and 0.4, respectively. It can be seen that the weight of user similarity decreased in the FSTI model, while that of item similarity increased to a certain extent. Hence, both models will perform more excellently under proper parameter settings.

\subsection{Effect of control parameter $\delta$}

For each dataset, the parameters $\alpha, \beta, z, \chi$ and $\theta$ were set to their optimal values, the first control parameter $s$ was fixed at 0.5 , and the second control parameter $\delta$ was adjusted in the interval $[0,1]$ by a step size of 0.1 . The effects of the adjustment on the FSTA and the FSTI are displayed in Figure 5(a) and Figure 5(b), respectively.

As shown in Figure 5, the optimal $\delta$ values of the FSTI on FilmTrust, Ciao and Epinions were 0.3, 0.1 and 0.3, respectively, revealing that, in the FSTI model, the truster is more important than the trustee of a user. Meanwhile, the optimal $\delta$ values of the FSTI on FilmTrust, Ciao and Epinions were 0.9, 0.3, and 0.8, respectively. It can be seen that the trusters of FilmTrust and Epinions are more important than those of Ciao. 


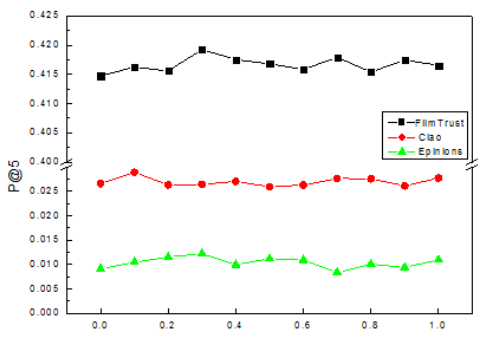

(a) FSTA

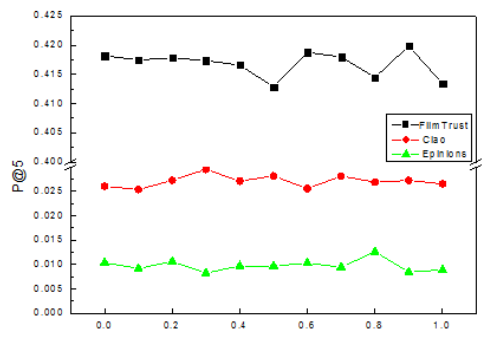

(b) FSTI

Figure 5: The effect of parameter $\delta$ on our approaches FSTA and FSTI in terms of precision at 5

\subsection{Comparison with other methods}

Table 4: The precisions of all algorithms on the three datasets (the precision of our approach is in bold).

\begin{tabular}{llllllll}
\hline Data & $\mathrm{d}$ & GBPR & MostPop & FISM & FST & FSTA & FSTI \\
\hline FilmTrust & 5 & 0.4124 & 0.4170 & 0.4171 & 0.4191 & $\mathbf{0 . 4 1 9 2}$ & $\mathbf{0 . 4 1 9 8}$ \\
& 10 & 0.3470 & 0.3503 & 0.3503 & 0.3514 & $\mathbf{0 . 3 5 2 5}$ & $\mathbf{0 . 3 5 3 0}$ \\
Ciao $\left(\times 10^{-1}\right)$ & 5 & 0.2228 & 0.2677 & 0.2704 & 0.2714 & $\mathbf{0 . 2 8 9 2}$ & $\mathbf{0 . 2 9 5 3}$ \\
& 10 & 0.1827 & 0.2142 & 0.2141 & 0.2174 & $\mathbf{0 . 2 2 4 5}$ & $\mathbf{0 . 2 3 3 7}$ \\
Epinions $\left(\times 10^{-1}\right)$ & 5 & 0.09353 & 0.1169 & 0.1147 & 0.1179 & $\mathbf{0 . 1 2 2 2}$ & $\mathbf{0 . 1 2 6 0}$ \\
& 10 & 0.07560 & 0.09171 & 0.09102 & 0.09187 & $\mathbf{0 . 1 0 4 1 0}$ & $\mathbf{0 . 1 0 9 6 3}$ \\
\hline
\end{tabular}

Table 5: The F1-measures of all algorithms on the three datasets (the precision of our approach is in bold)

\begin{tabular}{llllllll}
\hline Data & $\mathrm{d}$ & GBPR & MostPop & FISM & FST & FSTA & FSTI \\
\hline FilmTrust & 5 & 0.4051 & 0.4095 & 0.4087 & 0.4099 & $\mathbf{0 . 4 1 0 3}$ & $\mathbf{0 . 4 1 0 7}$ \\
& 10 & 0.4458 & 0.4518 & 0.4516 & 0.4521 & $\mathbf{0 . 4 5 3 3}$ & $\mathbf{0 . 4 5 3 4}$ \\
Ciao $\left(\times 10^{-1}\right)$ & 5 & 0.2063 & 0.2436 & 0.2495 & 0.2523 & $\mathbf{0 . 2 5 2 7}$ & $\mathbf{0 . 2 8 4 1}$ \\
& 10 & 0.2292 & 0.2662 & 0.2687 & 0.2720 & $\mathbf{0 . 2 7 4 3}$ & $\mathbf{0 . 2 8 2 9}$ \\
Epinions $\left(\times 10^{-1}\right)$ & 5 & 0.1103 & 0.1298 & 0.1307 & 0.1330 & $\mathbf{0 . 1 4 1 9}$ & $\mathbf{0 . 1 4 8 1}$ \\
& 10 & 0.1111 & 0.1305 & 0.1315 & 0.1328 & $\mathbf{0 . 1 3 8 1}$ & $\mathbf{0 . 1 4 6 7}$ \\
\hline
\end{tabular}

The $P @ N$ and $F 1 @ N$ of the FSTA and the FSTI were compared with those of the contrastive recommendation methods. The comparison results are listed in Tables 4 and 5. Overall, our approach outperformed the other methods under the same parameter configurations.

In the FilmTrust dataset, the MostPop achieved better results than the FISM. A possible reason lies in the consumption of the hot items in the dataset. In both Ciao and Epinions, the FISM outshined the MostPop, indicating the advantage of the factorized similarity model over the GBPR and BPR methods.

Moreover, the FSTA typically performed better than the methods, e.g. the FST, that integrates user similarity, item similarity and mutual trust. Note that the user rating matrix has a positive impact on the calculation of mutual trust, which comes from the trustee and the truster, respectively.

Finally, the comparison between the FSTA and the FST shows the influence of social impact on ranking performance. Our experimental parameters $\alpha, \beta, z, \chi$ and $\theta$ were adjusted only in one group. Better results can be obtained by adjusting the parameter set. 


\section{Conclusion}

Based on dynamically weighted trust relationship, a new mutual trust measurement method was put forward according to the difference in mutual trust strength. The method takes account of the direct and indirect trust relationships between users, thus improving the recommendation accuracy. Using the topology of social network, a new mutual influence measurement model was developed based on trust relationship. Considering both direct and indirect mutual influences, the proposed model makes full use of the implicit information in trust relationship.

Two new hybrid top-N recommendation models, involving user similarity, item similarity, mutual trust and mutual influence, were designed to solve the data binarity and sparsity of mutual trust, and used to explore the existence of social network users, identify potential trust relationships, and set up a mutual trust network. Our models were proved efficient through repeated comparative verifications on three standard datasets, namely, FilmTrust, Epinions and Ciao. The future research will explore the other factors that affect the mutual trust and further improve the performance of our models.

\section{Acknowledgement}

The work is supported by the National Science Foundation of China under Grant(No.61473108), and the National Experimental Teaching Demonstration Center of Computer Science in China. We thank Guibing Guo and his team for enlightening discussions.

\section{Bibliography}

[1] Callebert, L.; Lourdeaux, D.; BarthĂ“"s, J.P. (2018). Collective activity and autonomous characters: trust-based decision-making system, Revue d'Intelligence Artificielle, 31(1-2), 153-181, 2018.

[2] Coste, B.; Ray, C.; Coatrieux, G. (2017). Trust modelling and measurements for the security

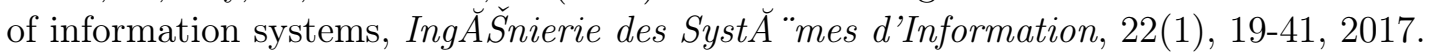

[3] Fang, H.; Bao, Y.; Zhang, J. (2014). Leveraging decomposed trust in probabilistic matrix factorization for effective recommendation, Twenty-Eighth AAAI Conference on Artificial Intelligence, 30-36, 2014.

[4] Guo, X.; Yin, S.; Zhang, Y.; Li, W.; He, Q. (2019). Cold start recommendation based on attribute-fused singular value decomposition, IEEE Access, 7, 11349-11359, 2019.

[5] Guo G.; Zhang J.; Yorke-Smith N (2016). A Novel Recommendation Model Regularized with User Trust and Item Ratings, IEEE Transactions on Knowledge $\& 3$ Data Engineering, 28(7), 1607-1620, 2016.

[6] Guo G.; Zhang J.; Zhu F. et al (2017). Factored similarity models with social trust for top-N item recommendation, Knowledge-Based Systems, 122, 17-25, 2017.

[7] Guo G(2019). List of Recommendation Data Sets, https://www.librec.net/datasets.html, 2011/6-2013/11.

[8] Han, Z.M.; Chen, Y.; Liu, W.; Yuan, B.H.; Li, M.Q.; Duan, D.G. (2017). Research on node influence analysis in social networks, Journal of Software, 28(1): 84-104, 2017. 
[9] Jamali, M.; Ester, M. (2010). A matrix factorization technique with trust propagation for recommendation in social networks, ACM Conference on Recommender Systems, 135-142, 2010 .

[10] Kabbur, S.; Ning, X.; Karypis, G. (2013). Fism: factored item similarity models for top-n recommender systems, Proceedings of the 19th ACM SIGKDD International Conference on Knowledge Discovery and Data Mining, 659-667, 2013.

[11] Li, W.; Ye, Z.; Xin, M.; Jin, Q. (2017). Social recommendation based on trust and influence in SNS environments, Multimedia Tools \& Applications, 76(9), 11585-11602, 2017.

[12] Moradi, P.; Ahmadian, S. (2015). A reliability-based recommendation method to improve trust-aware recommender systems, Expert Systems with Applications, 42(21), 7386-7398, 2015.

[13] Pan, W.; Chen, L. (2013). GBPR: group preference based Bayesian personalized ranking for one-class collaborative filtering, Twenty-Third International Joint Conference on Artificial Intelligence, 2691-2697.

[14] Pan, Y.; He, F.; Yu, H. (2018). Social recommendation algorithm using implicit similarity in trust, Chinese Journal of Computers, 41(1), 65-81, 2018.

[15] Rendle, S.; Freudenthaler, C.; Gantner, Z.; Schmidt-Thieme, L. (2009). BPR: Bayesian personalized ranking from implicit feedback, Proceedings of the twenty-fifth conference on uncertainty in artificial intelligence, 452-461, 2009.

[16] Tang, J.; Gao, H.; Liu, H.; Sarmas, A.D. (2012). eTrust: Understanding trust evolution in an online world, Proceedings of the 18th ACM SIGKDD International Conference on Knowledge Discovery and Data Mining, 253-261.

[17] Wu, M.X.; Dong, L.S.; Jie, Z.Y.; Hu, X. (2015). Research on social recommender systems, Journal of Software, (6), 1356-1372, 2015.

[18] Wang, M.; Ma, J. (2016). A novel recommendation approach based on users' weighted trust relations and the rating similarities, Soft Computing, 20(10), 3981-3990, 2016.

[19] Wang, Q.; Wang, J.H. (2015). Collaborative filtering recommendation algorithm combining trust mechanism with user preferences, Computer Engineering and Applications, 51(10), 261-265, 2015.

[20] Yang, X.; Guo, Y.; Liu, Y.; Steck, H. (2014). A survey of collaborative filtering based social recommender systems, Computer Communications, 41, 1-10, 2014.

[21] Yao, Q.; Shi, R.; Zhou, C.; Wang, P.; Guo, L. (2016). Topic-aware social influence minimization, Proceedings of the 24th International Conference on World Wide Web, 139-140 2015 .

[22] Zhao, F.; Guo, Y. (2016). Improving Top-N recommendation with heterogeneous loss, International Joint Conference on Artificial Intelligence, 2378-2384, 2016.

[23] Zhao, H.Y.; Hou, J.D.; Chen, Q.K. (2015). Collaborative filtering recommendation algorithm combining time weight and trust relationship, Application Research of Computers, 32(12), 3565-3568, 2015. 
[24] Zhang, D.; Sui, J.; Gong, Y. (2017). Large scale software test data generation based on collective constraint and weighted combination method, Tehnicki Vjesnik, 24(4), 1041-1050, 2017.

[25] Zhang, J.; Tang, J.; Li, J.; Liu, Y.; Xing, C.X. (2015). Who influenced you? Predicting retweet via social influence locality, ACM Transactions on Knowledge Discovery from Data (TKDD), 9(3), 25, 2015. 